\title{
A SIX-CHANNEL MICROSTRIP DIPLEXER FOR MULTI- SERVICE WIRELESS COMMUNICATION SYSTEMS
}

\author{
Farhad Fouladi ${ }^{1}$ - Abbas Rezaei ${ }^{1^{*}}$
}

${ }^{1}$ Department of Electrical Engineering, Kermanshah University of Technology, Kermanshah, Iran

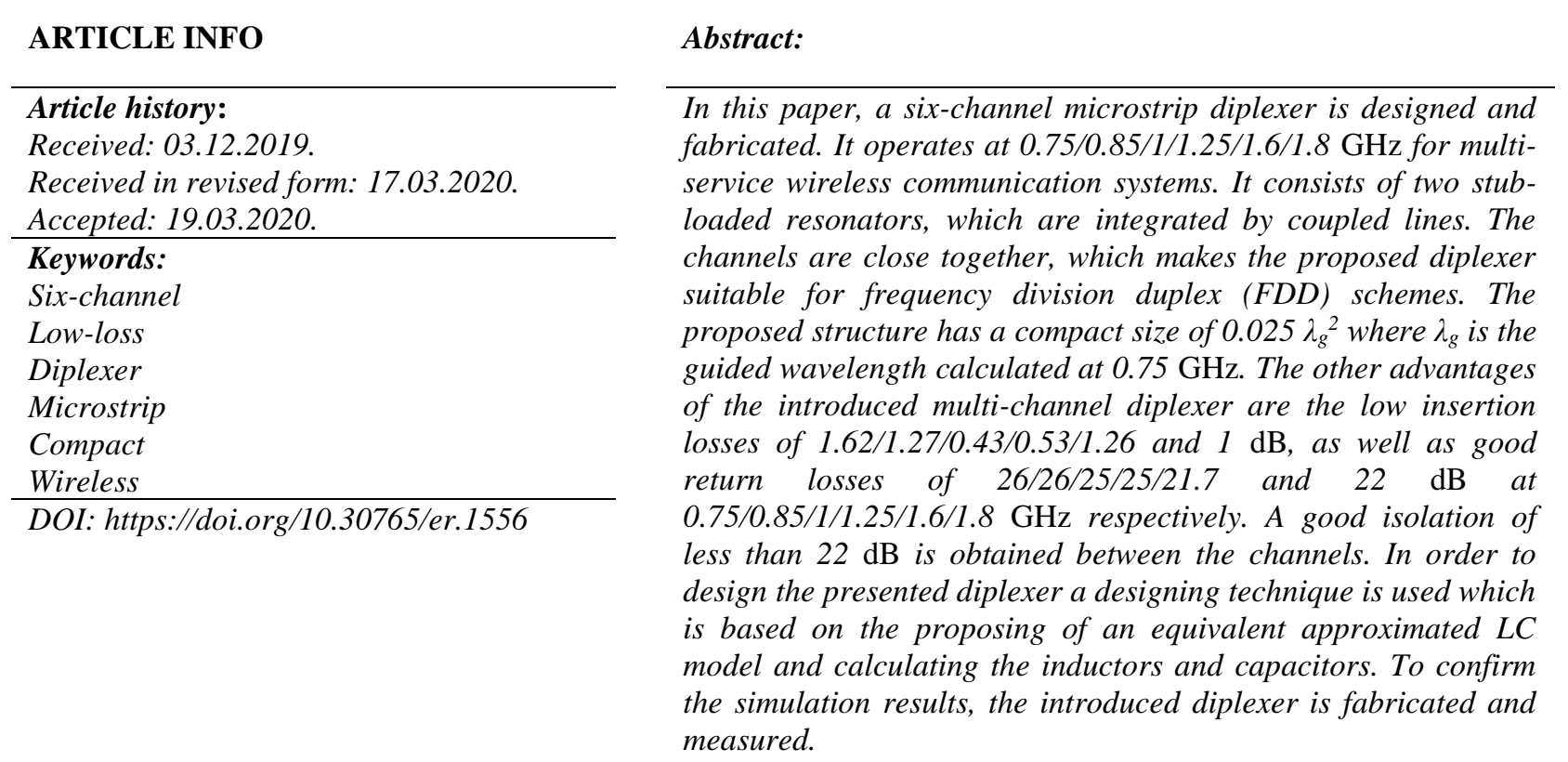

\section{Introduction}

Diplexers with multiple bandpass channels are essential components in multi-service and multi-band communication systems. They are used for controlling the transmitted and received signals in the RF front ends systems. Specifications of a well-designed multi-channel diplexer are low loss, high isolation and compact geometry. The compact microstrip structures with lightweight and two-dimensional geometry are appropriate components to design microwave multi-channel diplexers and multiplexers. Therefore, several diplexers, triplexers and multiplexers have been designed using various microstrip structures [1-9]. Sixchannel diplexers in $[1,2]$, four-channel diplexers in [3, 4], two-channel diplexers in [5, 6], and eightchannel diplexer in [7] were introduced. In [1], magnetically quarter wavelength coupled step impedance resonators by grounded via hole were used to obtain six passbands at 1.575/1.8/2.4/3.5/5.2/5.8 GHz. The proposed structure in [1] provides relatively compact size but the multiplexers and diplexers in [2-9] take up a lot of space. A new method of harmonic suppression was applied to achieve six passbands at 10/12/19/21/32/35 (GHz) in [2]. However, there are large insertion and return losses in its passbands. In [3], coupled hairpin resonators, in [4] quad-mode stub-loaded resonators, in [5] simple coupled lines, in [6] triangular open loop resonator and in [7] coupled loops with step impedance structures were utilized to design multi-channel diplexers. Due to the difficulty of designing multi-channel diplexers $[1-4,7]$, they have been less designed than single-band diplexers [3, 4] and multiplexers [8, 9]. The microstrip triplexer presented in [8] operates at 3.3/ 3.89/4.56 GHz. It is large in size and has a high insertion and return losses. In [9], a microstrip multiplexer with six passbands at six ports was realized. Designing the multiplexer in [9] was carried out by a combination of several lumped inductors and microstrip coupled lines.

\footnotetext{
* Corresponding author

E-mail address: a.rezaee@kut.ac.ir
} 
In [10], the design of a dual-frequency microstrip diplexer is investigated using the microstrip cells analysis and coupled lines components. In [11], a compact and high-isolation six-channel microstrip diplexer is presented using two pairs of multimode resonators. In [12], a compact tunable microstrip eight-channel multiplexer is investigated using dual-mode stub-loaded resonators. In [13], a new quad-channel diplexer operated at $2.45 \mathrm{GHz}, 4.2 \mathrm{GHz}, 3.5 \mathrm{GHz}$ and $5.2 \mathrm{GHz}$ is proposed, which is composed of folded defected stepped impedance resonators (DSIRs) and feeding lines. In [14], two quad-channel bandpass filters have been designed based on a novel circular multi-mode resonator.

In this paper, a compact six-channel diplexer is introduced, which operates at the resonance frequencies of 0.75/0.85/1/1.25/1.60/1.8 GHz for frequency division duplex (FDD) scheme [15-16] and multi-band wireless applications. It consists of new stub-loaded U-shape resonators, which are integrated by the coupled lines. The proposed diplexer is compact with low insertion loss that is designed to satisfy the requirements of multi-band communication systems. The steps of designing are organized as following:

First, an approximated LC circuit of a basic microstrip structure is proposed; then the values of lumped elements are calculated according to the transmission line theory. Next, the EM simulation results are compared to the simulation results of the LC model. This comparison partially verifies the approximated LC circuit. After that, the input impedance of the proposed basic structure is checked out to obtain the resonance modes. Finally, the compatibility of the obtained resonance conditions with the calculated lumped elements is presented, which verifies the designing methodology.

\section{Designing method}

The block diagram in Figure 1a shows how our six-channel diplexer works. Port 2 is connected to a transmitter, port 3 is connected to a receiver and port 1 is common between the transmitter and receiver. The signals are received and sent through port 1 by an antenna. Figure $1 \mathrm{~b}$ depicts a basic configuration to implement the presented block diagram. It consists of three microstrip-coupled lines, which integrate two resonators. Resonator 1 and coupled lines create three passbands at $0.75 \mathrm{GHz}, 1 \mathrm{GHz}$ and $1.6 \mathrm{GHz}$ while resonator 2 and coupled lines create the other passbands at $0.85 \mathrm{GHz}, 1.25 \mathrm{GHz}$ and $1.8 \mathrm{GHz}$. We assume that the basic structure of the resonators 1 and 2 are similar. The proposed resonators are composed of the microstrip stub loaded coupled lines.

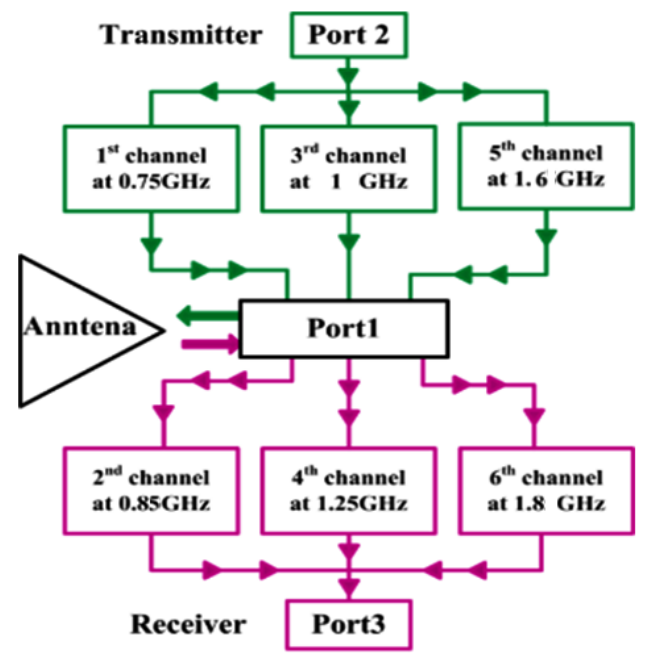

(a)

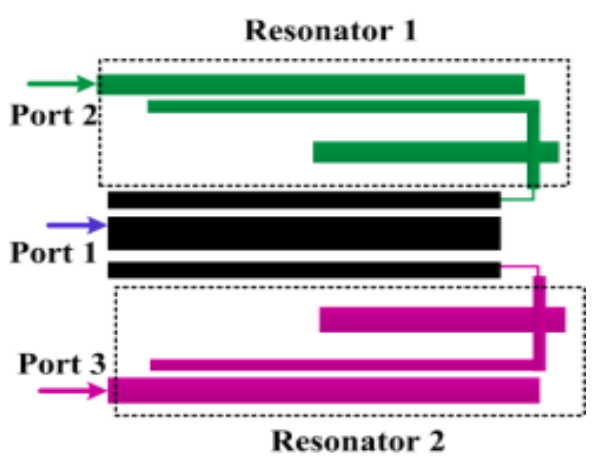

(b)

Figure 1. (a) Block diagram of the proposed six-channel diplexer (b) proposed basic configuration to implement the block diagram.

In Figure 2, an equivalent LC circuit of the proposed basic structure is outlined on the basic layout configuration. The corresponding LC circuit of each microstrip cell is drawn itself. The open ends of the stubs are replaced by the capacitor Co. Several inductors and capacitors model the coupled lines. An inductor is related to a half line and capacitors show the coupling between lines. These capacitors are $C_{R}$ for 
resonators and $\mathrm{C}_{\mathrm{C}}$ for the middle coupled lines. $\mathrm{L}_{\mathrm{S} 1}$ and $\mathrm{L}_{\mathrm{S} 2}$ are the inductors of the open stubs connected to the Resonator 1 and Resonator 2, respectively.

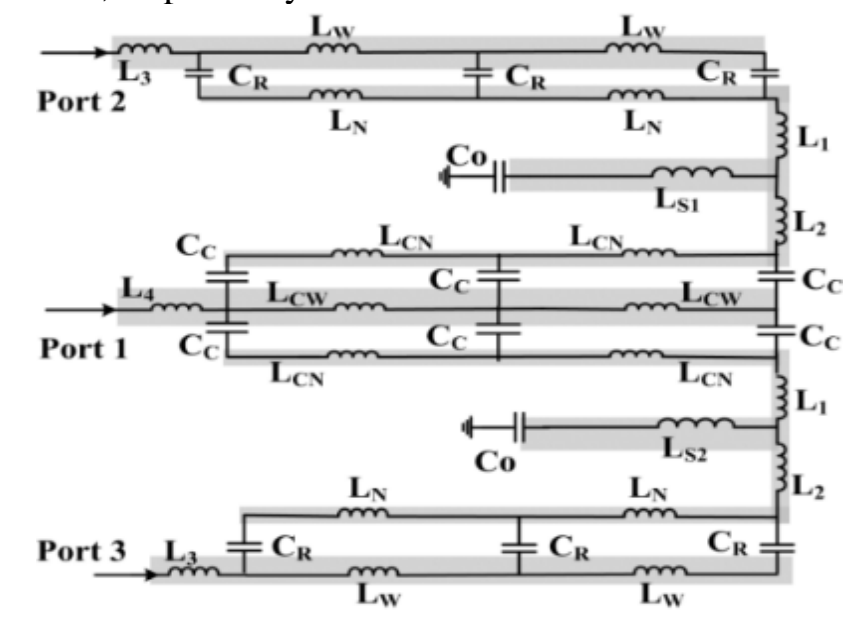

Figure 2. Equivalent LC circuit of the proposed basic structure.

Figure 2 depicts an approximate equivalent circuit. Since bents have a significant effect at the frequencies above $10 \mathrm{GHz}$, we ignored them [17]. Meanwhile, the equivalent circuit is a lossless passive network. In addition, the equivalent model of coupled lines is replaced approximately. Since we use an approximated model, we ignored a capacitor considering the dielectric effect of the substrate to have easier calculations.

The layout of the Resonator 1 and coupled lines connected to port 1 is presented in Figure 3a, which creates three passbands. This is a symmetric structure that obtains three channels. The resonator 2 and the middle coupled lines must create other channels. The equivalent LC circuit of Figure 3a is shown in Figure $3 \mathrm{~b}$. According to the presented dimensions in Figure 3a and based on the transmission line theory, the values of lumped elements can be calculated. They will be obtained using the input impedance of a transmission line $Z_{\text {in. }}$ A microstrip cell with the characteristic impedance " $Z c$ ", thickness " $h$ ", effective dielectric constant " $\varepsilon_{r e}$ ", width " $w$ " and length " $l$ " has the input impedance $\mathrm{Z}_{\text {in }}$ that can be calculated at a frequency of " $f$ " as follows [18]:

$$
\begin{aligned}
& Z_{\text {in }}=j Z c \tan \left(\frac{\pi l f \sqrt{\varepsilon_{r e}}}{150}\right), \text { where }: \varepsilon_{r e}=\left(\frac{\varepsilon_{r}+1}{2}+\frac{\varepsilon_{r}-1}{2 \sqrt{1+12 \frac{h}{w}}}\right) \\
& \text { and } Z c=\frac{120 \pi}{\left[\frac{w}{h}+1.393+0.677 \ln \left(1.44+\frac{w}{h}\right)\right] \sqrt{\varepsilon_{r e}}}
\end{aligned}
$$

Based on our preliminary conditions $h=0.508 \mathrm{~mm}, \varepsilon_{r}=3.15$ and the widths are $0.6 \mathrm{~mm}$ or $1.2 \mathrm{~mm}$. For $w=0.6 \mathrm{~mm}$, the characteristic impedance and effective dielectric constant are $68.98 \Omega$ and 2.37, respectively. Similarly, for $w=1.2 \mathrm{~mm}$, the characteristic impedance and effective dielectric constant are $46.74 \Omega$ and 2.51 , respectively. The lumped inductors are obtained by calculating $Z_{\text {in. }}$ For each inductor " $L$ " we can write $L=Z_{\text {in }} /(j 2 \pi f L)$, where $Z_{\text {in }}$ gets different values for each microstrip cell. However, for the shunt stub, $L_{S 1}$ will be calculated by $Z_{i n}=j 2 \pi f L_{S 1}+1 /(j 2 \pi f C o)$. Meanwhile, we assumed $C o=1.3 \mathrm{pF}$ to calculate $L_{S I}$. The inductors of the LC circuit are calculated and listed in Table 1. According to Equation (1), the inductors have different values at each resonance frequencies. Based on the EM simulation, the results obtained from the layout in Figure 3a, this basic microstrip structure resonates at three different frequencies i.e. 0.96/1.9/2.9 GHz. Therefore, the lumped inductors are calculated at these frequencies. Some values in Table 1 are negative that represent the capacitive modes and we ignore them. The same values in Table 1 are due to the symmetric structure of basic layout. Using the calculated data in Table 1, the LC circuit presented in Figure $3 \mathrm{~b}$ is simulated. Then this simulation is compared to the EM simulator as shown in Figure $3 \mathrm{c}$ and Figure $3 \mathrm{~d}$. The 
EM and schematic simulation results are approximately similar, which verifies the proposed LC model. Similar to the layout results, the schematic results are obtained by advanced design system (ADS) software. The open stubs $\mathrm{L}_{1}$ and $\mathrm{L}_{2}$ resonate at lower frequency. The capacitors of couplings $\left(\mathrm{Cc}\right.$ and $\left.\mathrm{C}_{\mathrm{R}}\right)$ are small to represent the coupling features so that they will be in $f F$. As shown in Figure $3 \mathrm{c}$, both EM and schematic simulation results are approximately similar. However, there are some differences such as the frequency shift around $0.5 \mathrm{GHz}$, which is due to the approximation of LC circuit.

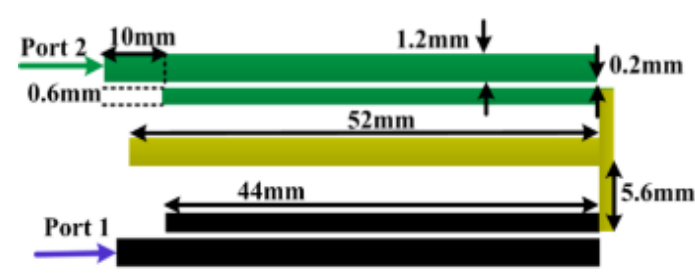

(a)

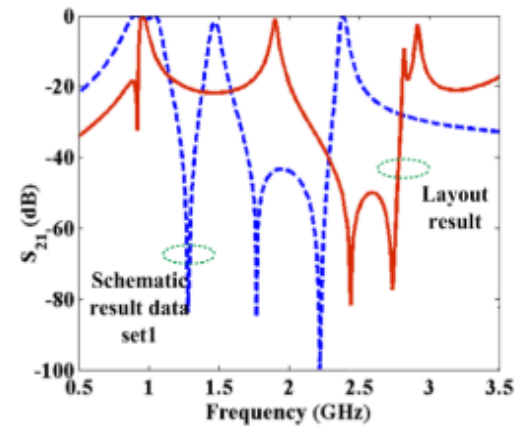

(c)

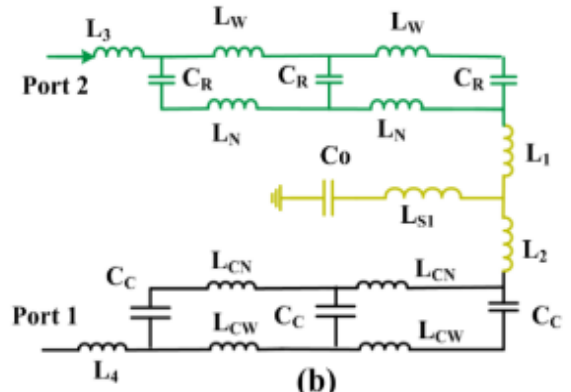

(b)

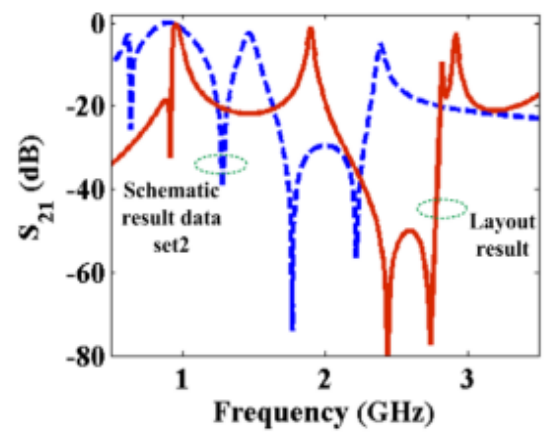

(d)

Figure 3. (a) Basic structure consisting of the Resonator 1 and coupled lines, (b) LC model of the proposed basic structure, (c) comparison of layout and schematic simulations for $L_{1}=L_{2}=0.48 n H, L_{3}=L_{4}=2.84 \mathrm{nH}$, $L_{S l}=6.03 \mathrm{nH}, C o=1.3 \mathrm{pf}, L_{W}=L_{C W}=20.52 \mathrm{nH}, L_{N}=L_{C N}=49.34 \mathrm{nH}$ and $C_{R}=C_{C}=220 \mathrm{fF}$,(d) comparison of layout and schematic simulations for $L_{1}=L_{2}=0.48 \mathrm{nH}, L_{3}=L_{4}=2.84 \mathrm{nH}, L_{S l}=6.03 \mathrm{nH}, C o=1.3 \mathrm{pf}, L_{W}=20.52$ $\mathrm{nH}, L_{N}=49.34 \mathrm{nH}, C_{R=} 220 \mathrm{fF}, L_{C W}=2.134 \mathrm{nH}, L_{C N}=2.159 \mathrm{nH}$ and $C_{C}=14.4 \mathrm{nf}$.

Table 1. Lumped element values calculated at three various frequencies (inductors are in $n H$ ).

\begin{tabular}{cccccccccc}
\hline \multicolumn{1}{c}{ Elements } & $\mathbf{L}_{\mathbf{1}}$ & $\mathbf{L}_{\mathbf{2}}$ & $\mathbf{L}_{\mathbf{3}}$ & $\mathbf{L}_{\mathbf{4}}$ & $\mathbf{L}_{\mathbf{S 1}}$ & $\mathbf{L}_{\mathbf{W}}$ & $\mathbf{L}_{\mathbf{N}}$ & $\mathbf{L}_{\mathbf{C W}}$ & $\mathbf{L}_{\mathbf{C N}}$ \\
\hline \hline Values at $0.96 \mathrm{GHz}$ & 0.48 & 0.48 & 0.989 & 0.989 & 6.03 & 2.134 & 2.159 & 2.134 & 2.159 \\
Values at $1.9 \mathrm{GHz}$ & 1.817 & 1.817 & 2.84 & 2.84 & 0.49 & 20.52 & 49.34 & 20.52 & 49.34 \\
Values at $2.9 \mathrm{GHz}$ & 1.9 & 1.9 & 3.67 & 3.67 & -25 & -4.23 & -7.36 & -4.23 & -7.36 \\
\hline \hline
\end{tabular}

Similarly, the elements of the LC model in Figure $3 \mathrm{~d}$ are $L_{1}=L_{2}=0.48 \mathrm{nH}, L_{3}=L_{4}=2.84 \mathrm{nH}, L_{\mathrm{S} 1}=6.03 \mathrm{nH}$, $C o=1.3 \mathrm{pf}, L_{\mathrm{W}}=20.52 \mathrm{nH}, L_{\mathrm{N}}=49.34 \mathrm{nH}, C_{\mathrm{R}}=220 \mathrm{fF}, L_{\mathrm{CW}}=2.134 \mathrm{nH}, L_{\mathrm{CN}}=2.159 \mathrm{nH}$ and $C_{\mathrm{C}}=14.4 \mathrm{nf}$. In order to create three passbands through the port 2 and port 1 , the input impedance between ports 1 and 2 can be zero. For the microstrip structure presented in Figure 3b, the total input impedance viewed from port 2 can be calculated using a simplified LC circuit. Figure 4 shows the steps of simplification to calculate the total input impedance $Z_{\text {total }}$, where $\omega$ is the angular frequency. Due to the symmetrical structure, first we assume $C_{\mathrm{R}}=C_{\mathrm{C}}=C, L_{\mathrm{W}}=L_{\mathrm{CW}}, L_{\mathrm{N}}=L_{\mathrm{CN}}, L_{2}=L_{1}=L \mathrm{a}$ and $L_{3}=L_{4}=L_{\mathrm{b}}$, then, we connect same potential nodes, which leads to reduce the LC circuit as shown in Figure 4. Finally, with a delta star transformation the LC circuit is simplified. Therefore, the $Z_{\text {total }}$ is calculated as follows:

$$
Z_{\text {total }}=0.5 j \omega L_{b}+Z_{2}+Z s+\frac{\left(0.5 j \omega L_{w}+Z_{3}\right) \times\left(Z_{1}+Z_{e}\right)}{0.5 j \omega L_{w}+Z_{3}+Z_{1}+Z_{e}}
$$


The values of $Z_{1}, Z_{2}, Z_{3}, Z$ s and $Z e$ are shown in Figure 4.
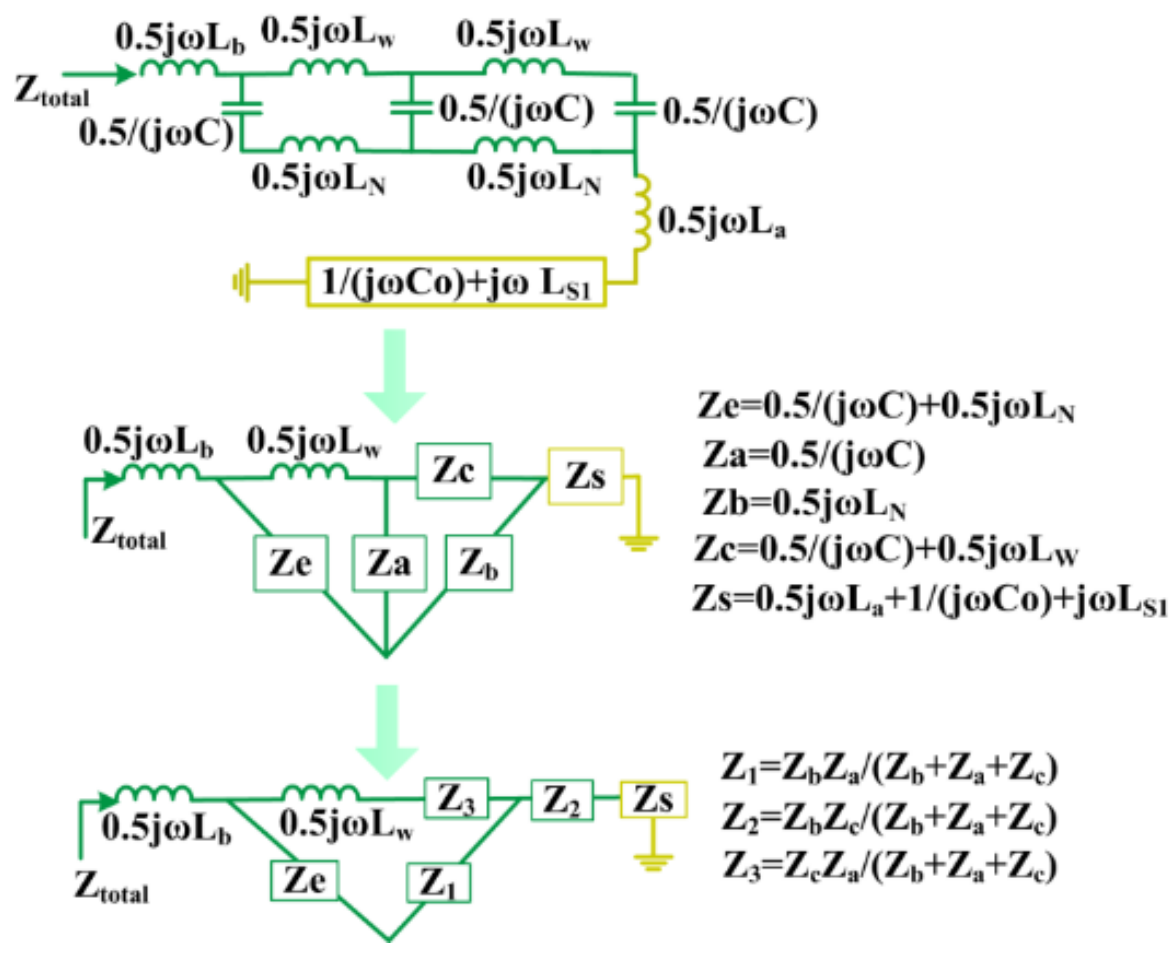

$\mathbf{Z}_{1}=\mathbf{Z}_{\mathrm{b}} \mathbf{Z}_{\mathrm{a}} /\left(\mathbf{Z}_{\mathrm{b}}+\mathbf{Z}_{\mathrm{a}}+\mathbf{Z}_{\mathrm{c}}\right)$
$\mathbf{Z}_{2}=\mathbf{Z}_{\mathrm{b}} \mathbf{Z}_{\mathrm{c}} /\left(\mathbf{Z}_{\mathrm{b}}+\mathbf{Z}_{\mathrm{a}}+\mathbf{Z}_{\mathrm{c}}\right)$
$\mathbf{Z}_{3}=\mathbf{Z}_{\mathrm{c}} \mathbf{Z}_{\mathrm{a}} /\left(\mathbf{Z}_{\mathrm{b}}+\mathbf{Z}_{\mathrm{a}}+\mathbf{Z}_{\mathrm{c}}\right)$

Figure 4. Simplification of the LC circuit to calculate $Z_{\text {total. }}$.

For the even mode, the resonance condition is achieved when $Z_{\text {total }}{ }^{-1}=0$. Therefore, we can write a resonance condition as follows:

$$
\begin{aligned}
& 0.5 j \omega L_{w}+Z_{3}+Z_{1}+Z_{e}=0 \Rightarrow 0.5 j \omega L_{w}+\frac{Z_{a} Z_{c}+Z_{b} Z_{a}}{Z_{b}+Z_{a}+Z_{c}}+ \\
& \frac{0.5}{j \omega C}+0.5 j \omega L_{N}=0 \Rightarrow \omega^{2} C\left(L_{w}+L_{N}\right)=\left[\frac{\left(3-2 \omega^{2} C\left(L_{w}+L_{N}\right)\right)}{2-\omega^{2} C\left(L_{w}+L_{N}\right)}\right] \Rightarrow \\
& \omega=\left\{\begin{array}{l}
\left(C\left(L_{w}+L_{N}\right)\right)^{-0.5} \\
3\left(C\left(L_{w}+L_{N}\right)\right)^{-0.5}
\end{array}\right.
\end{aligned}
$$

According to Equation (3), coupled lines create two resonance frequencies. In this case, the third passband channel should be created by the condition of odd mode resonance frequency. If we select the calculated inductors as $L_{\mathrm{W}}=2.134 \mathrm{nH}$ and $L_{\mathrm{N}}=2.159 \mathrm{nH}$, for the resonance frequency of $f=0.96 \mathrm{GHz}$ from Equation (3), the coupling capacitor $\mathrm{C}$ will be $C=C_{\mathrm{C}}=C_{\mathrm{R}}=169.48 \mathrm{nf}$ but for $f=1.9 \mathrm{GHz}, L_{\mathrm{W}}=20.52 \mathrm{nH}$ and $L_{\mathrm{N}}=49.34 \mathrm{nH}$ the coupling capacitor will be $300.43 \mathrm{fF}$, which is calculated by $\omega=3\left[C\left(L_{w}+L_{N}\right)\right]^{-0.5}$. This value of $C$ is so near to that so we selected to compare the EM simulator with the LC simulation results. This verifies the design methodology. The layout configuration of the proposed diplexer is designed based on the geometrical structure of Figure 2 and explained designing method. The proposed six-channel diplexer is presented in Figure 5. Additional optimizations are carried out to tune the resonance frequencies and miniaturization. For example, the shunt direct stubs are changed to spirals to save the overall size. In Figure 6 , current density distribution of the proposed diplexer is shown. 


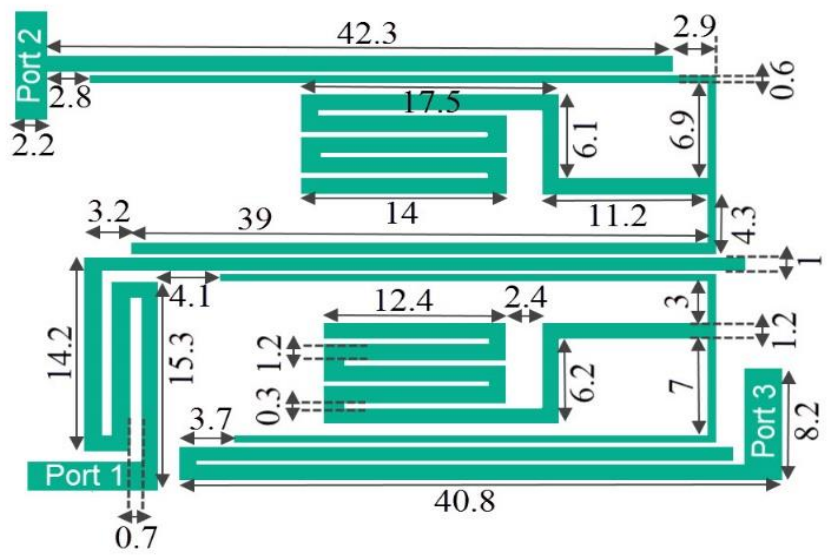

Figure 5. Dimensions of proposed diplexer (in $\mathrm{mm}$ ).

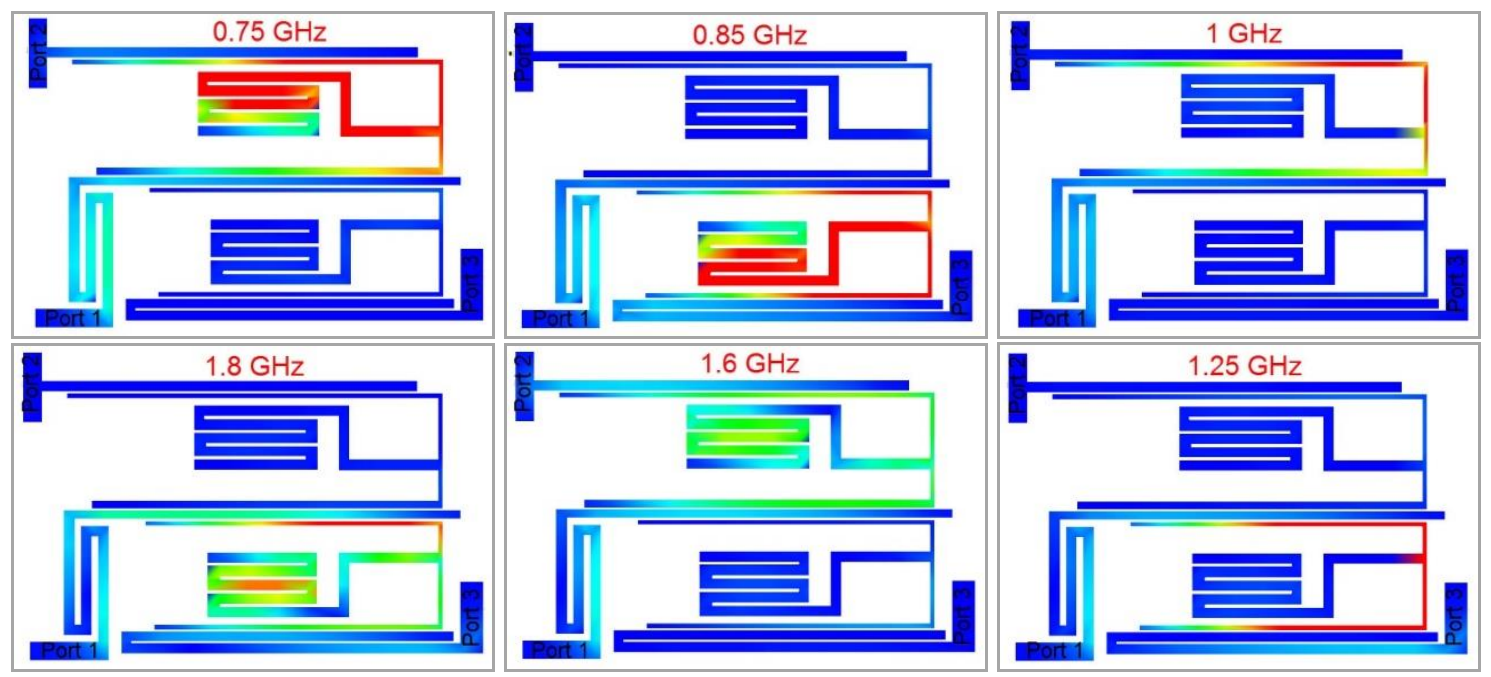

Figure 6. Current density distribution of the proposed diplexer.

\section{Results and discussion}

The designed multi-channel diplexer is simulated by the ADS full wave EM simulator and fabricated on the substrate RT_Duroid_5880 with the dielectric constant 2.22, loss tangent $=0.0008$ and thickness of 0.508 $\mathrm{mm}$. Finally, it is measured by the Agilent Network Analyser N5230A. The overall size of the proposed structure is $52.1 \mathrm{~mm} \times 35.1 \mathrm{~mm}\left(0.19 \lambda_{\mathrm{g}} \times 0.13 \lambda_{\mathrm{g}}\right)$, where $\lambda_{\mathrm{g}}$ is the guided wavelength calculated at $0.75 \mathrm{GHz}$. Figures $7 \mathrm{a}$ and $7 \mathrm{~b}$ depict the simulated and measured frequency responses consisting of the proposed diplexer. A photograph of the fabricated diplexer and $S_{11}$ are shown in Figure 7c. According to the obtained results, the proposed diplexer has a frequency response at the central frequencies of $0.75 \mathrm{GHz}, 1 \mathrm{GHz}$ and $1.6 \mathrm{GHz}$ (port 2) and $0.85 \mathrm{GHz}, 1.25 \mathrm{GHz}$ and1.8 GHz (port3). At the central frequencies, the insertion losses are $1.62 \mathrm{~dB}, 1.27 \mathrm{~dB}, 0.43 \mathrm{~dB}, 0.53 \mathrm{~dB}, 1.26 \mathrm{~dB}$ and $1 \mathrm{~dB}$ and the return losses are $26 \mathrm{~dB}, 26 \mathrm{~dB}, 25$ $\mathrm{dB}, 25 \mathrm{~dB}, 21.7 \mathrm{~dB}$ and $22 \mathrm{~dB}$, respectively. The performance of the proposed six-channel diplexer is compared with the six-channel diplexers, four-channel diplexers, two-channel diplexers, eight-channel diplexers and multiplexers. The comparison results are summarized in Table 3. According to Table 3, the realized diplexer not only has a minimum circuit size, but also good insertion losses and reasonable return losses at all passbands achieved. 


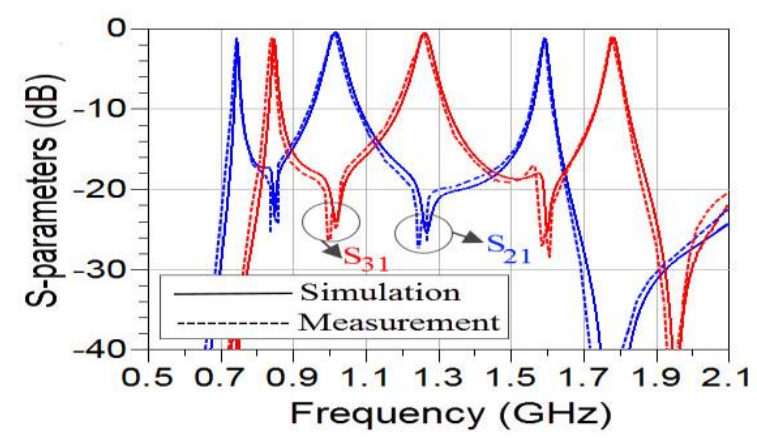

(a)

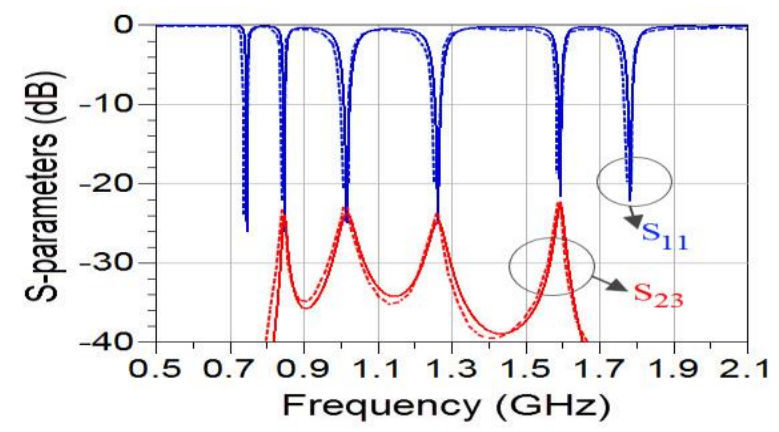

(b)

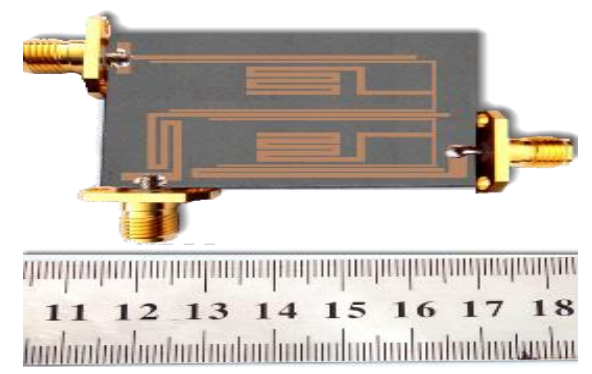

(c)

Figure 7. (a) Simulated and measured $S_{21}$ and $S_{31}(b)$ simulated and measured $S_{11}$ and $S_{23}$ (narrowband response), (c) fabricated diplexer.

Table 3. Comparison Table ( ${ }^{*} C$ : Number of Channels and P: Number of ports).

\begin{tabular}{|c|c|c|c|c|}
\hline \multirow{2}{*}{ References } & \multirow{2}{*}{$\mathbf{C} / \mathbf{P}^{*}$} & \multirow{2}{*}{ Resonance frequencies $(\mathbf{G H z})$} & Insertion losses (dB) & \multirow{2}{*}{$\operatorname{Size}\left(\lambda \mathrm{g}^{2}\right)$} \\
\hline & & & Return Loss (dB) & \\
\hline \multirow{2}{*}{ This Work } & \multirow{2}{*}{$6 / 3$} & \multirow{2}{*}{ 0.75/0.85/1/1.25/1.60/1.8 } & $1.62 / 1.27 / 0.43 / 0.53 / 1.26 / 1$ & \multirow{2}{*}{0.025} \\
\hline & & & $26 / 26 / 25 / 25 / 21.7 / 22$ & \\
\hline \multirow{2}{*}{ [1] } & \multirow{2}{*}{$6 / 3$} & \multirow{2}{*}{$1.57 / 1.8 / 2.4 / 3.5 / 5.2 / 5.8$} & $1.5 / 1.1 / 1.5 / 1.2 / 2 / 2$ & \multirow{2}{*}{0.027} \\
\hline & & & Better than $20 \mathrm{~dB}$ at all channels & \\
\hline \multirow{2}{*}[2]{} & \multirow{2}{*}{$6 / 3$} & \multirow{2}{*}{$10 / 12 / 19 / 21 / 32 / 35$} & $2.2 / 3.1 / 3.2 / 2 / 2.8 / 3$ & \multirow{2}{*}{4} \\
\hline & & & Better than $10 \mathrm{~dB}$ at all channels & \\
\hline \multirow{2}{*}{ [3] } & \multirow{2}{*}{$4 / 3$} & \multirow{2}{*}{$0.9 / 1.5 / 2.4 / 3.5$} & $2.02 / 1.56 / 2.08 / 2.52$ & \multirow{2}{*}{0.042} \\
\hline & & & Better than $13 \mathrm{~dB}$ at all channels & \\
\hline \multirow{2}{*}{ [4] } & \multirow{2}{*}{$4 / 3$} & \multirow{2}{*}{$0.9 / 1.2 / 1.5 / 1.8$} & $1.36 / 1.5 / 1.3 / 1.6$ & \multirow{2}{*}{0.049} \\
\hline & & & $17.5 / 18.3 / 19.6 / 20.2$ & \\
\hline \multirow{2}{*}[5]{} & \multirow{2}{*}{$2 / 3$} & \multirow{2}{*}{$1.8 / 2.4$} & $2.2 / 2.1$ & \multirow{2}{*}{0.064} \\
\hline & & & $11.9 / 12.0$ & \\
\hline \multirow{2}{*}{ [6] } & \multirow{2}{*}{$2 / 3$} & \multirow{2}{*}{$2.3 / 2.55$} & $1.5 / 1.3$ & \multirow{2}{*}{0.102} \\
\hline & & & Better than $21 \mathrm{~dB}$ at all channels & \\
\hline & & & $2.1 / 2.3 / 2.4 / 2.6 / 2.3 / 2.2 / 2.8 / 2.3$ & \\
\hline$[7]$ & $8 / 3$ & $0.9 / 1.2 / 1.5 / 1.8 / 2.1 / 2.4 / 2.7 / 3$ & Better than $13 \mathrm{~dB}$ at all channel & 0.1 \\
\hline [Q] & $3 / 4$ & $32 / 380 / 456$ & $2.2 / 2.3 / 2.3$ & 0275 \\
\hline [8] & $3 / 4$ & $3.3 / 3.89 / 4.56$ & Better than $14 \mathrm{~dB}$ at all channels & $0.2 / 5$ \\
\hline & & & $2.13 / 2.3 / 2.2 / 2.1 / 2.08 / 2.76$ & \\
\hline [9] & $6 / 7$ & 0.8/1.1/1.4/1.7/2/2.3 & Better than $11 \mathrm{~dB}$ at all channels & 0.048 \\
\hline$[101$ & $2 / 3$ & $236 / 4$ & $0.2 / 0.4$ & 0006 \\
\hline$[10]$ & $2 / 3$ & $2.36 / 4$ & $15 / 11$ & 0.096 \\
\hline & & & $1.8 / 1.9 / 1 / 1.5 / 2.1 / 2.6$ & \\
\hline [11] & $6 / 3$ & $1.8 / 2.4 / 3 / 3.84 .7 / 5.8$ & $11.7 / 28.4 / 16.8 / 16.9 / 10.1 / 15.8$ & 0.057 \\
\hline [12] & $8 / 8$ & $085 / 105 / 13 / 15 / 165 / 185 / 205 / 23$ & $2.7 / 2.4 / 2.4 / 3 / 3.4 / 3.8 / 4.2 / 4.6$ & 0105 \\
\hline$[12]$ & & & Better than 15 & \\
\hline [13] & $2 / 4$ & $2.2+3$ & $0.22 / 0.3 / 1.32 / 2.12$ & 088 \\
\hline [13] & $2 / 4$ & 2.45/4.2/3.5/5.2 & Better than 10 & 0.88 \\
\hline
\end{tabular}




\section{Conclusion}

This paper presented a microstrip six-channel diplexer with a compact size of $0.19 \lambda_{\mathrm{g}} \times 0.13 \lambda_{\mathrm{g}}$, low insertion losses and good return losses. It is designed to operate at $0.75 \mathrm{GHz}, 1 \mathrm{GHz}, 1.6 \mathrm{GHz}, 0.85 \mathrm{GHz}$, $1.25 \mathrm{GHz}$ and $1.8 \mathrm{GHz}$ for multi-band and multi-service wireless networks. The proposed structure consists of two stub loaded U-shape resonators which are integrated by the coupled lines. This diplexer in comparison to several types of diplexers and multiplexers had the minimum size. Meanwhile, a challenge is in the number of passbands where due to higher design difficulty of six-channel diplexers, they have been less designed. Another advantage of the proposed multi-channel diplexer was its close channels, which make it appropriate for the frequency division duplex (FDD) applications.

\section{References}

[1] Chen Y-W., Wu H-W., Dai Z-J., Su Y-K., Design of Compact Six-Channel Diplexer. IEEE Microwave and Wireless Components Letters. 26(10) (2016), 792-794.

[2] Hong S., Chang K., A 10-35 GHz Six-Channel Microstrip Multiplexer for Wide-Band Communication Systems. IEEE Transaction on Microwave Theory and Technique. 54(4) (2006), 1370-1378.

[3] Hsu K-W, Hung W-C, Tu W-H., Design of Four-Channel Diplexer Using Distributed Coupling Technique. Microwave and Optical Technology Letters, 58(1) (2016), 166-170.

[4] Lin L, Zhao Y-T, Wu B, Liang C-H., Design of Compact Quad-Channel Diplexer Using Quad-Mode Stub-Loaded Resonators. Progress In Electromagnetics Research Letters. 51 (2015), 87-93.

[5] Yan J-M, Zhou H-Y, Cao L-Z., Compact Diplexer Using Microstrip Half- and Quarter-Wavelength Resonators. IET Electronics Letters. 52(19) (2016), 1613-1615.

[6] Salehi MR, Keyvan S, Abiri E, Noori L., Compact Microstrip Diplexer Using New Design of Triangular Open Loop Resonator for $4 G$ Wireless Communication Systems. International Journal of Electronics and Communications (AEÜ). 70(7) (2016), 961-969.

[7] Tu W-H, Hung W-C., Microstrip Eight-Channel Diplexer with Wide Stopband. Microwave and Wireless Components Letters. 24(11) (2014), 742-744.

[8] CW T, Chen MG, Packaged Microstrip Triplexer with Star-Junction Topology. Electronics Letters. 48 (2012), 699-701.

[9] Tu W-H, Hsu K-W., Design of Sext-Band Bandpass Filter and Sextaplexer Using Semilumped Resonators for System in a Package. IEEE Transaction on Components Packaging and Manufacturing Technology. 5(2) (2015), 265-273.

[10] Noori L, Rezaei A., Design of a Microstrip Dual-Frequency Diplexer Using Microstrip Cells Analysis and Coupled Lines Components. International Journal of Microwave and Wireless Technologies. 9(7) (2017), 1467-1471.

[11] Li Q, Zhang Y, Six-channel diplexer with compact size and high isolation, Electronics letters, 53(17) (2017), 1205-1207.

[12] Chen C-F, Tseng B-H, Wang G-Y, Li G-J, Compact microstrip eight-channel multiplexer with independently switchable passbands, IET Microwaves, antennas \& propagation, 12(6) (2018), 10261033.

[13] Zhu A, Zhou H, Chen J, Li J, Compact Quad-Channel Diplexer Using Defected Stepped Impedance Resonators, Progress In Electromagnetics Research Letters, 80 (2018), 127-133.

[14] Noori L, Rezaei A, Design of microstrip wide stopband quad-band bandpass filters for multi-service communication systems, AEU - International Journal of Electronics and Communications, 81 (2017), 136-142.

[15] Peng. H, Chiang. Y., Microstrip diplexer constructed with new types of dual-mode ring filters, IEEE Microwave Wireless Compon Lett. 25 (2015), 7-9.

[16] Rezaei. A, Noori. L., Mohammadi. H, Design of a novel compact microstrip diplexer with low insertion loss, Microwave and Optical Technology Letters, 59(7), (2017), 1672-1676.

[17] Rezaei. A, Noori. L, S. M. Hosseini., Novel microstrip branch-line coupler with low phase shift for WLANs, Analog Integrated Circuits and Signal Processing, 98 (2019), 377-383.

[18] J. S Hong and M. J. Lancaster, Microstrip Filters for RF/Microwave Applications, John Wiley \& Sons, 2001. 